\title{
Atopobium rimae
}

National Cancer Institute

\section{Source}

National Cancer Institute. Atopobium rimae. NCI Thesaurus. Code C114244.

A species of strictly anaerobic, Gram-positive, rod or elliptical shaped bacteria arranged in single element, pairs or chains, in the phylum Actinobacteria. This species is nonmotile, non-spore forming, negative for indole production and nitrate reduction, ferments glucose to yield lactic acid, acetic acid and formic acid, and does not produce gas from glucose fermentation. A. rimae is found in the normal ging ival flora but can cause odontogenic infection, chronic periodontitis and bacteremia in humans. 